\title{
The Limit Behavior of a Stochastic Logistic Model with Individual Time-Dependent Rates
}

\author{
Yilun Shang ${ }^{1,2}$ \\ ${ }^{1}$ Institute for Cyber Security, University of Texas at San Antonio, San Antonio, TX 78249, USA \\ ${ }^{2}$ Singapore University of Technology and Design, Singapore 138682 \\ Correspondence should be addressed to Yilun Shang; shylmath@hotmail.com
}

Received 28 January 2013; Revised 21 April 2013; Accepted 7 May 2013

Academic Editor: William E. Fitzgibbon

Copyright (c) 2013 Yilun Shang. This is an open access article distributed under the Creative Commons Attribution License, which permits unrestricted use, distribution, and reproduction in any medium, provided the original work is properly cited.

We investigate a variant of the stochastic logistic model that allows individual variation and time-dependent infection and recovery rates. The model is described as a heterogeneous density dependent Markov chain. We show that the process can be approximated by a deterministic process defined by an integral equation as the population size grows.

\section{Introduction}

The stochastic logistic model, also called the endemic SIS model in the epidemiological context, was first discussed by Weiss and Dishon [1]. This model describes the evolution of an infection in a fixed population of size $n$ by a continuoustime Markov chain for the number of infected individuals $Y^{n}$. The state space is $\{0,1, \ldots, n\}$, and the transition rates are given by

$$
\begin{gathered}
Y^{n} \longrightarrow Y^{n}+1 \quad \text { at rate } n^{-1} \lambda Y^{n}\left(n-Y^{n}\right), \\
Y^{n} \longrightarrow Y^{n}-1 \quad \text { at rate } \mu Y^{n},
\end{gathered}
$$

where $\lambda$ and $\mu$ are the infection rate of susceptibles and the recovery rate of infectives, respectively. This simple model has found applications in a variety of fields, including population biology, metapopulation ecology, chemistry, and physics. Properties such as mean epidemic size [2,3], mean extinction time $[1,4,5]$, and quasi-stationary distributions [6-8] have been extensively studied under different initial conditions.

The stochastic logistic model has an interesting limit property that it can be approximated by deterministic differential equations. In particular, based on Theorem 3.1 of [9], the rescaled process $n^{-1} Y^{n}$ converges in probability uniformly on finite time intervals to the solution of an ordinary differential equation. The issue of differential equation approximations for stochastic processes traces back to the pioneer work of Kurtz $[9,10]$, where deterministic limit of pure jump density-dependent Markov processes was add-ressed via Trotter type approximation theorems. Recently, McVinish and Pollett [11] show that a deterministic limit process can be established for a stochastic logistic model with individual variation, where the coefficients of the transition rates of the Markov chain can vary with the nodes. We refer the interested reader to a comprehensive survey [12] for numerous sufficient conditions for this type of convergence.

In this paper, along the above line of study, we investigate a variant of the stochastic logistic model where both individual variation and time-dependent infection and recovery rates are allowed. Specifically, we consider a continuous-time Markov chain $X^{n}=\left(X_{1}^{n}, \ldots, X_{n}^{n}\right)$ on the state space $\{0,1\}^{n}$ with transition rates given by

$$
\begin{gathered}
X^{n} \longrightarrow X^{n}+e_{i}^{n} \quad \text { at rate } \lambda_{i, t}\left(1-X_{i}^{n}\right) f\left(n^{-1} \sum_{j=1}^{n} g_{j}\left(X_{j}^{n}\right)\right), \\
X^{n} \longrightarrow X^{n}-e_{i}^{n} \quad \text { at rate } \mu_{i, t} X_{i}^{n},
\end{gathered}
$$

where $i=1,2, \ldots, n, \lambda_{i, t}, \mu_{i, t} \in \mathbb{R}_{+}$, and $e_{i}^{n}$ is the $n$-dimensional vector whose $i$ th entry is 1 , and other entries are 0 . Let $X_{i}^{n}=0$ represent that individual $i$ is susceptible and $X_{i}^{n}=1$ 
represent that individual $i$ is infected. Then $Y^{n}=\sum_{i=1}^{n} X_{i}^{n}$ is equivalent to the stochastic logistic model by setting $\lambda_{i, t}=\lambda$, $\mu_{i, t}=\mu, f(x)=x$, and $g_{j}(x)=x$ for all $i, j$, and $t$.

The above model (2) can be viewed as a generalization of that treated in [11] in twofolds. Firstly, the infection and recovery rates are time varying, incorporating realistic scenarios where the infection and recovery capacities may change over time [13]. As such, the transition rates of the Markov chain are explicitly time dependent, making the previously obtained sufficient conditions no longer applicable.

Secondly, the introduction of functions $g_{j}(x)$ accommodates needed flexibility for some applications. Indeed, since $X_{j}^{n} \in\{0,1\}$, the linear form $g_{j}(x)=\kappa_{j} x$ used in [11] implies that no contribution can be made by susceptible individuals. This is only a rough approximation. For one thing, susceptible individuals aware of a disease in their proximity can take measures (such as wearing masks, avoiding public places, and frequent hand washing) to reduce their susceptibility, which in turn affect the epidemic dynamics dramatically [14, 15]. For another, the epidemic progression strongly depends on the contact patterns between susceptible and infected individuals, especially in the network context. Identifying individual role is an interesting and demanding task [16]. In the present framework, each node $j$ applies individual contribution $g_{j}(0)$ (and $g_{j}(1)$ ), indicating the underlying interaction structure/strength among individuals.

The rest of the paper is organized as follows. We state the main result in Section 2 and provide the proof in Section 3.

\section{The Result}

In what follows, we assume naturally that $g_{j}(1) \geq g_{j}(0) \geq 0$ for all $j$. We will show that the stochastic logistic model (2) converges weakly to the solution of an integral equation as the population size $n \rightarrow \infty$.

Let $(S, \mathscr{B})$ be a measurable space with $S \subseteq \mathbb{R}_{+}^{2}$ and $\mathscr{B}$ the Borel $\sigma$-algebra on $S$. Denote by $C_{b}(S)$ the set of bounded, continuous functions on $S$. Let $\Omega$ be the set of $\sigma$ finite measures on $S$. For $n \in \mathbb{N}$ and $h \in C_{b}(S)$, we define the measure-valued nonrandom process $\left\{\sigma_{t}^{n}, t \in \mathbb{R}_{+}\right\}$and the measure-valued Markov process $\left\{\rho_{t}^{n}, t \in \mathbb{R}_{+}\right\}$by

$$
\begin{aligned}
\sigma_{t}^{n}(h): & =\int_{S} h(\mu, \lambda) \sigma_{t}^{n}(\mathrm{~d} \mu, \mathrm{d} \lambda), \\
& =n^{-1} \sum_{i=1}^{n}\left(g_{i}(1)-g_{i}(0)\right) h\left(\mu_{i, t}, \lambda_{i, t}\right), \\
\rho_{t}^{n}(h): & =\int_{S} h(\mu, \lambda) \rho_{t}^{n}(\mathrm{~d} \mu, \mathrm{d} \lambda) \\
& =n^{-1} \sum_{i=1}^{n}\left(g_{i}(1)-g_{i}(0)\right) X_{i, t}^{n} h\left(\mu_{i, t}, \lambda_{i, t}\right) .
\end{aligned}
$$

For some set $A$, let $D\left(\mathbb{R}_{+}, A\right)$ be the set of right-continuous functions with left-hand limits mapping $\mathbb{R}_{+}$to $A$. Thus, for $n \in \mathbb{N}, \sigma^{n} \in D\left(\mathbb{R}_{+}, \Omega\right)$ and the sample paths of $\rho^{n}$ belong to $D\left(\mathbb{R}_{+}, \Omega\right)$, since the sample paths of $X^{n}$ belong to $D\left(\mathbb{R}_{+},\{0,1\}^{n}\right)$ (application of Theorem 16.16 in $[17$, page 316]).

We assume that the following assumptions hold.

(A1) $n^{-1} \sum_{i=1}^{n} g_{i}(0) \rightarrow \bar{g}<\infty$, and $\sup _{n} \bar{g}_{1, n}<\infty$, where $\bar{g}_{a, n}=n^{-a} \sum_{i=1}^{n}\left(g_{i}(1)-g_{i}(0)\right)^{a}$ for $a \in \mathbb{N}$.

(A2) $\sigma_{t}^{n} \stackrel{d}{\rightarrow} \sigma_{t}$ uniformly for $t \in \mathbb{R}_{+}$, and $\rho_{0}^{n} \stackrel{d}{\rightarrow} \rho_{0}$ in $\Omega$ as $n \rightarrow \infty$, where $\sigma_{t}$ and $\rho_{0}$ are nonrandom measures, and $\stackrel{d}{\rightarrow}$ means weak convergence (i.e., $\lim _{n \rightarrow \infty} \sigma_{t}^{n}(h)=\sigma_{t}(h)$, and $\rho_{0}^{n}(h)$ converges to $\rho_{0}(h)$ in distribution as $n \rightarrow \infty$ for all $h \in C_{b}(S)$ [17, page 316]).

(A3) $S$ is bounded in $\mathbb{R}_{+}^{2}$.

(A4) $f: \mathbb{R}_{+} \rightarrow \mathbb{R}_{+}$is Lipschitz continuous.

(A5) $\lambda_{i, t}$ and $\mu_{i, t}$ as functions of $t$ are piecewise constant for $i=1, \ldots, n$.

Theorem 1. If assumptions (A1)-(A5) hold, then the measurevalued Markov process $\rho_{t}^{n}$ converges weakly to a measurevalued nonrandom process $\rho_{t} \in D\left(\mathbb{R}_{+}, \Omega\right)$, that is,

$$
\rho^{n} \stackrel{d}{\longrightarrow} \rho,
$$

as $n \rightarrow \infty$, where $\rho$ is the unique solution of

$$
0=\rho_{t}(h)-\rho_{0}(h)-\int_{0}^{t} G \rho_{s}(h) \mathrm{d} s,
$$

with

$$
\begin{gathered}
\rho_{t}(h)=\int_{S} h(\mu, \lambda) \rho_{t}(\mathrm{~d} \mu, \mathrm{d} \lambda) \\
G \rho_{t}(h)=\int_{S} \lambda h(\mu, \lambda) f\left(\int_{S} \rho_{t}\left(\mathrm{~d} \mu^{\prime}, \mathrm{d} \lambda^{\prime}\right)+\bar{g}\right) \sigma_{t}(\mathrm{~d} \mu, \mathrm{d} \lambda) \\
-\int_{S} \lambda h(\mu, \lambda) f\left(\int_{S} \rho_{t}\left(\mathrm{~d} \mu^{\prime}, \mathrm{d} \lambda^{\prime}\right)+\bar{g}\right) \rho_{t}(\mathrm{~d} \mu, \mathrm{d} \lambda) \\
-\int_{S} \mu h(\mu, \lambda) \rho_{t}(\mathrm{~d} \mu, \mathrm{d} \lambda) .
\end{gathered}
$$

Let $L^{1}(S)$ be the space of $\sigma_{t}$-integrable functions on $S$. We have the following corollary.

Corollary 2. Suppose that $\rho$ is the unique solution to (5). Then there exists a unique function

$$
\begin{aligned}
\varphi: \mathbb{R}_{+} & \longrightarrow L^{1}(S) \\
t & \longmapsto \varphi_{t}
\end{aligned}
$$

with $0 \leq \varphi_{t} \leq 1$ such that

$$
\rho_{t}(A)=\int_{A} \varphi_{t}(\mu, \lambda) \sigma_{t}(\mathrm{~d} \mu, \mathrm{d} \lambda)
$$

for all Borel set $A \in S$, and

$$
\begin{aligned}
& \varphi_{t}=\varphi_{0}+\int_{0}^{t}( \lambda f\left(\int_{S} \varphi_{s}\left(\mu^{\prime}, \lambda^{\prime}\right) \sigma_{s}\left(\mathrm{~d} \mu^{\prime}, \mathrm{d} \lambda^{\prime}\right)+\bar{g}\right) \\
&\left.\times\left(1-\varphi_{s}\right)-\mu \varphi_{s}\right) \mathrm{d} s .
\end{aligned}
$$




\section{Proofs}

In this section, Theorem 1 will be proved through a series of lemmas by tightness and uniqueness arguments [18]. The idea of proofs is similar to that in [11], and we include the complete proofs here, not only for the convenience of the reader but also to convince the reader that the results do hold in our setting.

Lemma 3. The sequence $\rho^{n}$ is tight in $D\left(\mathbb{R}_{+}, \Omega\right)$.

Proof. Recall that [17, Theorem 16.27, page 324] $\rho^{n}$ is tight in $D\left(\mathbb{R}_{+}, \Omega\right)$ if and only if $\rho_{t}^{n}(h)$ is tight in $D\left(\mathbb{R}_{+}, \mathbb{R}\right)$ for every $h \in C_{b}(S)$. According to Aldous's tightness criterion [18, Theorem 16.10, page 178], the tightness of $\rho_{t}^{n}(h)$ holds, if the following two conditions are satisfied.

(A) For each $\varepsilon>0, \eta>0$, and $r \geq 1$, there exist a $\delta_{0}>0$ and an $n_{0} \in \mathbb{N}$ such that if $\tau_{n}$ is a discrete $\mathscr{F}_{t}^{n}$-stopping time satisfying $\tau_{n} \leq r$, then

$$
\sup _{n \geq n_{0}} \sup _{\delta \leq \delta_{0}} P\left(\left|\rho_{\tau_{n}}^{n}(h)-\rho_{\tau_{n}+\delta}^{n}(h)\right| \geq \eta\right) \leq \varepsilon .
$$

(B) For each $r>0$,

$$
\lim _{a \rightarrow \infty} \limsup _{n \rightarrow \infty} P\left(\sup _{t \leq r} \rho_{t}^{n}(h)>a\right)=0 .
$$

To show (A), we express the generator of $X^{n}$ by

$$
\begin{aligned}
G_{n} l(x)=\sum_{i=1}^{n}\left(l\left(x+e_{i}^{n}\right)-l(x)\right) \lambda_{i, t}\left(1-x_{i}\right) \\
\quad \times f\left(n^{-1} \sum_{j=1}^{n} g_{j}\left(x_{j}\right)\right) \\
+\sum_{i=1}^{n}\left(l\left(x-e_{i}^{n}\right)-l(x)\right) \mu_{i, t} x_{i}
\end{aligned}
$$

for all real-valued functions $l(x)$ with $x=\left(x_{1}, \ldots, x_{n}\right) \in$ $\{0,1\}^{n}$. Hence, using (3), we obtain

$$
\begin{aligned}
& G_{n} \rho_{t}^{n}(h) \\
& =\int_{S} \lambda h(\mu, \lambda) f\left(\int_{S} \rho_{t}^{n}\left(\mathrm{~d} \mu^{\prime}, \mathrm{d} \lambda^{\prime}\right)\right. \\
& \left.+n^{-1} \sum_{j=1}^{n} g_{j}(0)\right) \sigma_{t}^{n}(\mathrm{~d} \mu, \mathrm{d} \lambda) \\
& -\int_{S} \lambda h(\mu, \lambda) f\left(\int_{S} \rho_{t}^{n}\left(\mathrm{~d} \mu^{\prime}, \mathrm{d} \lambda^{\prime}\right)\right. \\
& \left.+n^{-1} \sum_{j=1}^{n} g_{j}(0)\right) \rho_{t}^{n}(\mathrm{~d} \mu, \mathrm{d} \lambda) \\
& -\int_{S} \mu h(\mu, \lambda) \rho_{t}^{n}(\mathrm{~d} \mu, \mathrm{d} \lambda) .
\end{aligned}
$$

Applying Dynkin's formula to $\rho_{t}^{n}(h)$ (see, e.g., [19, Proposition 1.7, page 162]), we have that

$$
M_{t}^{n}(h)=\rho_{t}^{n}(h)-\rho_{0}^{n}(h)-\int_{0}^{t} G_{n} \rho_{s}^{n}(h) \mathrm{d} s
$$

is a martingale with respect to the filtration $\mathscr{F}_{t}^{n}=\sigma\left\{X_{i, s}^{n}, 0 \leq\right.$ $s \leq t\}$, and $M_{0}^{n}(h)=0$. Moreover, $M_{t}^{n}(h)$ is square integrable. The condition (A) holds if for each $\varepsilon>0, \eta>0$, and $r \geq 1$, there exist a $\delta_{0}>0$ and an $n_{0} \in \mathbb{N}$ such that for any discrete $\mathscr{F}_{t}^{n}$-stopping time satisfying $\tau_{n} \leq r$,

$$
\begin{aligned}
& \sup _{n \geq n_{0}} \sup _{\delta \leq \delta_{0}} P\left(\left|\int_{\tau_{n}}^{\tau_{n}+\delta} G_{n} \rho_{s}^{n}(h) \mathrm{d} s\right| \geq \eta\right) \leq \varepsilon, \\
& \sup _{n \geq n_{0}} \sup _{\delta \leq \delta_{0}} P\left(\left|M_{\tau_{n}+\delta}^{n}(h)-M_{\tau_{n}}^{n}(h)\right| \geq \eta\right) \leq \varepsilon .
\end{aligned}
$$

To see (15), we note that

$$
\begin{aligned}
& \left|G_{n} \rho_{t}^{n}(h)\right| \\
& \leq \int_{S} \lambda h(\mu, \lambda) f\left(\int_{S} \rho_{t}^{n}\left(\mathrm{~d} \mu^{\prime}, \mathrm{d} \lambda^{\prime}\right)+n^{-1} \sum_{j=1}^{n} g_{j}(0)\right) \\
& \times \sigma_{t}^{n}(\mathrm{~d} \mu, \mathrm{d} \lambda) \\
& +\int_{S} \lambda h(\mu, \lambda) f\left(\int_{S} \rho_{t}^{n}\left(\mathrm{~d} \mu^{\prime}, \mathrm{d} \lambda^{\prime}\right)+n^{-1} \sum_{j=1}^{n} g_{j}(0)\right) \\
& \times \rho_{t}^{n}(\mathrm{~d} \mu, \mathrm{d} \lambda) \\
& +\int_{S} \mu h(\mu, \lambda) \rho_{t}^{n}(\mathrm{~d} \mu, \mathrm{d} \lambda) \\
& \leq \int_{S} h(\mu, \lambda)\left(2 \lambda f\left(\bar{g}_{1, n}+n^{-1} \sum_{j=1}^{n} g_{j}(0)\right)+\mu\right) \\
& \times \sigma_{t}^{n}(\mathrm{~d} \mu, \mathrm{d} \lambda) \\
& \leq \bar{g}_{1, n} \\
& \times \sup _{(\mu, \lambda) \in S}\left\{h(\mu, \lambda)\left(2 \lambda f\left(\bar{g}_{1, n}+n^{-1} \sum_{j=1}^{n} g_{j}(0)\right)+\mu\right)\right\},
\end{aligned}
$$

by using (3). It follows from Markov's inequality and (17) that

$$
\begin{aligned}
& P\left(\left|\int_{\tau_{n}}^{\tau_{n}+\delta} G_{n} \rho_{s}^{n}(h) \mathrm{d} s\right| \geq \eta\right) \\
& \leq \eta^{-1} E\left(\int_{\tau_{n}}^{\tau_{n}+\delta}\left|G_{n} \rho_{s}^{n}(h)\right| \mathrm{d} s\right) \\
& \leq \eta^{-1} \delta \bar{g}_{1, n} \\
& \quad \times \sup _{(\mu, \lambda) \in S}\left\{h(\mu, \lambda)\left(2 \lambda f\left(\bar{g}_{1, n}+n^{-1} \sum_{j=1}^{n} g_{j}(0)\right)+\mu\right)\right\}
\end{aligned}
$$


From assumptions (A1) and (A4), we obtain $\sup _{n} f\left(\bar{g}_{1, n}+\right.$ $\left.n^{-1} \sum_{j=1}^{n} g_{j}(0)\right)<\infty$. Therefore, we can find $\delta_{0}$ and $n_{0}$ such that (15) is satisfied by using the assumptions (A1), (A3), (A4), and the fact that $h \in C_{b}(S)$.

To prove (16), we need to introduce the quadratic variation process $\left[M^{n}(h)\right]$ for $M^{n}(h)$. Since $M_{t}^{n}(h)$ is a square integrable martingale, it follows from Proposition 6.1 in [19, page 79] that $\left(M^{n}(h)\right)^{2}-\left[M^{n}(h)\right]$ is also a martingale. By using Markov's inequality and martingale properties, we obtain

$$
\begin{aligned}
& P\left(\left|M_{\tau_{n}+\delta}^{n}(h)-M_{\tau_{n}}^{n}(h)\right| \geq \eta\right) \\
& \leq \eta^{-2} E\left(\left(M_{\tau_{n}+\delta}^{n}(h)-M_{\tau_{n}}^{n}(h)\right)^{2}\right) \\
& =\eta^{-2} E\left(E\left(\left(M_{\tau_{n}+\delta}^{n}(h)-M_{\tau_{n}}^{n}(h)\right)^{2} \mid \mathscr{F}_{\tau_{n}}^{n}\right)\right) \\
& =\eta^{-2}\left(E\left(\left(M_{\tau_{n}+\delta}^{n}(h)\right)^{2}\right)+E\left(\left(M_{\tau_{n}}^{n}(h)\right)^{2}\right)\right. \\
& \left.\quad-2 E\left(M_{\tau_{n}}^{n}(h) E\left(M_{\tau_{n}+\delta}^{n}(h) \mid \mathscr{F}_{\tau_{n}}^{n}\right)\right)\right) \\
& =\eta^{-2}\left(E\left(\left(M_{\tau_{n}+\delta}^{n}(h)\right)^{2}\right)-E\left(\left(M_{\tau_{n}}^{n}(h)\right)^{2}\right)\right) \\
& =\eta^{-2}\left(E\left(\left(M_{\tau_{n}+\delta}^{n}(h)\right)^{2}-\left[M^{n}(h)\right]_{\tau_{n}+\delta}\right)\right. \\
& \quad+E\left(\left[M^{n}(h)\right]_{\tau_{n}+\delta}\right) \\
& \left.\quad-E\left(\left(M_{\tau_{n}}^{n}(h)\right)^{2}-\left[M^{n}(h)\right]_{\tau_{n}}\right)-E\left(\left[M^{n}(h)\right]_{\tau_{n}}\right)\right) \\
& =\eta^{-2}\left(E\left(\left[M^{n}(h)\right]_{\tau_{n}+\delta}-\left[M^{n}(h)\right]_{\tau_{n}}\right)\right) .
\end{aligned}
$$

Applying Dynkin's formula to $\left(\rho_{t}^{n}(h)\right)^{2}$ similarly as in the derivation of (14), we have that

$$
\begin{aligned}
\widetilde{M}_{t}^{n}(h)= & \left(\rho_{t}^{n}(h)\right)^{2}-\left(\rho_{0}^{n}(h)\right)^{2} \\
& -2 \int_{0}^{t} \rho_{s}^{n}(h) G_{n} \rho_{s}^{n}(h) \mathrm{d} s-n^{-1} \int_{0}^{t} \widetilde{G}_{n} \rho_{s}^{n}(h) \mathrm{d} s
\end{aligned}
$$

is a martingale, where

$$
\begin{aligned}
& \widetilde{G}_{n} \rho_{t}^{n}(h) \\
& =\int_{S} \lambda h^{2}(\mu, \lambda) f\left(\int_{S} \rho_{t}^{n}\left(\mathrm{~d} \mu^{\prime}, \mathrm{d} \lambda^{\prime}\right)+n^{-1} \sum_{j=1}^{n} g_{j}(0)\right) \\
& \quad \times \widetilde{\sigma}_{t}^{n}(\mathrm{~d} \mu, \mathrm{d} \lambda) \\
& \quad-\int_{S} \lambda h^{2}(\mu, \lambda) f\left(\int_{S} \rho_{t}^{n}\left(\mathrm{~d} \mu^{\prime}, \mathrm{d} \lambda^{\prime}\right)+n^{-1} \sum_{j=1}^{n} g_{j}(0)\right) \\
& \quad \times \tilde{\rho}_{t}^{n}(\mathrm{~d} \mu, \mathrm{d} \lambda) \\
& \quad-\int_{S} \mu h^{2}(\mu, \lambda) \tilde{\rho}_{t}^{n}(\mathrm{~d} \mu, \mathrm{d} \lambda),
\end{aligned}
$$

with

$$
\begin{aligned}
& \int_{S} h(\mu, \lambda) \tilde{\sigma}_{t}^{n}(\mathrm{~d} \mu, \mathrm{d} \lambda) \\
& =n^{-1} \sum_{i=1}^{n}\left(g_{i}(1)-g_{i}(0)\right)^{2} h\left(\mu_{i, t}, \lambda_{i, t}\right), \\
& \int_{S} h(\mu, \lambda) \tilde{\rho}_{t}^{n}(\mathrm{~d} \mu, \mathrm{d} \lambda) \\
& \quad=n^{-1} \sum_{i=1}^{n}\left(g_{i}(1)-g_{i}(0)\right)^{2} X_{i, t}^{n} h\left(\mu_{i, t}, \lambda_{i, t}\right) .
\end{aligned}
$$

By using (14) and (20), we obtain for any $0 \leq s<t$,

$$
\begin{aligned}
\left(\rho_{t}^{n}(h)\right. & \left.-\rho_{s}^{n}(h)\right)^{2} \\
= & \left(\rho_{t}^{n}(h)\right)^{2}-\left(\rho_{s}^{n}(h)\right)^{2}-2 \rho_{s}^{n}(h)\left(\rho_{t}^{n}(h)-\rho_{s}^{n}(h)\right) \\
= & \widetilde{M}_{t}^{n}(h)-\widetilde{M}_{s}^{n}(h)+n^{-1} \int_{s}^{t} \widetilde{G}_{n} \rho_{r}^{n}(h) \mathrm{d} r \\
& -2 \rho_{s}^{n}(h)\left(M_{t}^{n}(h)-M_{s}^{n}(h)\right) \\
& +2 \int_{s}^{t} \rho_{r}^{n}(h) G_{n} \rho_{r}^{n}(h) \mathrm{d} r \\
& -2 \rho_{s}^{n}(h) \int_{s}^{t} G_{n} \rho_{r}^{n}(h) \mathrm{d} r .
\end{aligned}
$$

It follows from (3) and the assumption (A5) that $\rho_{t}^{n}(h)$ has piecewise constant sample paths, and $\int_{0}^{t} G_{n} \rho_{s}^{n}(h) \mathrm{d} s$ is a continuous finite variation process. Therefore, (14), (23), and the fact $\widetilde{M}_{0}^{n}(h)=0$ imply that

$$
\begin{aligned}
{\left[M^{n}(h)\right]_{t}=} & {\left[\rho^{n}(h)\right]_{t} } \\
= & \widetilde{M}_{t}^{n}(h)-2 \int_{0}^{t} \rho_{s}^{n}(h) d M_{s}^{n}(h) \\
& +n^{-1} \int_{0}^{t} \widetilde{G}_{n} \rho_{s}^{n}(h) \mathrm{d} s .
\end{aligned}
$$

From (19), (24), and martingale properties, we obtain

$$
\begin{aligned}
& P(\left.\left|M_{\tau_{n}+\delta}^{n}(h)-M_{\tau_{n}}^{n}(h)\right| \geq \eta\right) \\
& \leq n^{-1} \eta^{-2} E\left(\int_{\tau_{n}}^{\tau_{n}+\delta} \widetilde{G}_{n} \rho_{s}^{n}(h) \mathrm{d} s\right) \\
& \leq n^{-1} \eta^{-2} \\
& \quad \quad \int_{\tau_{n}}^{\tau_{n}+\delta} \int_{S} h^{2}(\mu, \lambda)\left(2 \lambda f\left(\bar{g}_{1, n}+n^{-1} \sum_{j=1}^{n} g_{j}(0)\right)+\mu\right) \\
& \leq n^{-1} \eta^{-2} \delta \bar{g}_{2, n} \\
& \quad \times \sup _{(\mu, \lambda) \in S}\left\{h^{2}(\mu, \lambda)\left(2 \lambda f\left(\bar{g}_{1, n}+n^{-1} \sum_{j=1}^{n} g_{j}(0)\right)+\mu\right)\right\} .
\end{aligned}
$$


The assumption (A1) implies that $\lim \sup _{i \rightarrow \infty}\left(g_{i}(1)-g_{i}(0)\right)<$ $\infty$, and hence $\sup _{n} \bar{g}_{2, n}<\infty$. Therefore, as in the derivation of (15), we can choose some $n_{0}$ and $\delta_{0}$ such that (16) is satisfied.

Now, the only thing remaining to verify is the condition (B). Since $h \in C_{b}(S)$ and the assumption (A3) holds, there exists a constant $C>0$ such that

$$
\sup _{t \leq r}\left|\rho_{t}^{n}(h)\right| \leq C \bar{g}_{1, n}
$$

for all $n \in \mathbb{N}$ and $r>0$. From Markov's inequality and the assumption (A1), we have

$$
\begin{aligned}
\limsup _{n \rightarrow \infty} P\left(\sup _{t \leq r} \rho_{t}^{n}(h)>a\right) & \leq \limsup _{n \rightarrow \infty} a^{-1} E\left(\sup _{t \leq r}\left|\rho_{t}^{n}(h)\right|\right) \\
& \leq a^{-1} C \sup _{n} \bar{g}_{1, n} \longrightarrow 0,
\end{aligned}
$$

as $a \rightarrow \infty$. The proof of Lemma 3 is complete.

Lemma 4. For any $h \in C_{b}(S), M^{n}(h) \stackrel{d}{\rightarrow} 0$ in $D\left(\mathbb{R}_{+}, \mathbb{R}\right)$ as $n \rightarrow \infty$.

Proof. By the corollary in [18, page 28], it is sufficient to show that $d_{\infty}^{0}\left(M^{n}(h), 0\right) \stackrel{p}{\rightarrow} 0$ as $n \rightarrow \infty$, where $\stackrel{p}{\rightarrow}$ means convergence in probability. Here, by definition, the space $D\left(\mathbb{R}_{+}, \mathbb{R}\right)$ is the so-called Skorohod space $D[0, \infty)$, and $d_{\infty}^{0}$ is the metric on it, which defines the Skorohod topology (see, e.g., [18, page 168]). Theorem 16.7 in [18, page 174] further implies that it is sufficient to show that $d_{t}^{0}\left(M^{n}(h), 0\right) \stackrel{p}{\rightarrow} 0$ for each $t \geq 0$. The metric $d_{t}^{0}$ generates the topology of Skorohod space $D[0, t]$ (see [18, pages 166 and 125] for definitions).

Since $d_{t}^{0}\left(M^{n}(h), 0\right) \leq \sup _{s \leq t}\left|M_{s}^{n}(h)\right|$, by Doob's martingale inequality, we have for any $\varepsilon>0$,

$$
\begin{aligned}
P\left(d_{t}^{0}\left(M^{n}(h), 0\right) \geq \varepsilon\right) & \leq P\left(\sup _{s \in[0, t]}\left|M_{s}^{n}(h)\right| \geq \varepsilon\right) \\
& \leq \varepsilon^{-2} E\left(\left(M_{t}^{n}(h)\right)^{2}\right) \\
& =\varepsilon^{-2} E\left[M^{n}(h)\right]_{t} \\
& =\varepsilon^{-2} n^{-1} E\left(\int_{0}^{t} \widetilde{G}_{n} \rho_{s}^{n}(h) \mathrm{d} s\right),
\end{aligned}
$$

where the first equality uses the fact that $\left(M_{t}^{n}(h)\right)^{2}-\left[M^{n}(h)\right]_{t}$ is a martingale with expectation 0 , and the second equality follows from (24). It then follows from the derivation in (25) that

$$
\lim _{n \rightarrow \infty} P\left(d_{t}^{0}\left(M^{n}(h), 0\right) \geq \varepsilon\right)=0,
$$

which concludes the proof of Lemma 4.

From Lemma 3, the sequence $\rho^{n}$ is tight, and then, there exists a weakly convergent subsequence $\rho^{n_{k}}$ in $D\left(\mathbb{R}_{+}, \Omega\right)$. We still denote it by $\rho^{n}$ in the next lemma for convenience.
Lemma 5. If $\rho^{n} \stackrel{d}{\rightarrow} \rho$ as $n \rightarrow \infty$, then for any $h \in C_{b}(S)$, $M^{n}(h) \stackrel{d}{\rightarrow} M(h)$ in $D\left(\mathbb{R}_{+}, \mathbb{R}\right)$ as $n \rightarrow \infty$, where

$$
M_{t}(h)=\rho_{t}(h)-\rho_{0}(h)-\int_{0}^{t} G \rho_{s}(h) \mathrm{d} s .
$$

Proof. For each $n \in \mathbb{N}$, define a random process $N^{n}(h)$ by

$$
N_{t}^{n}(h)=\rho_{t}^{n}(h)-\rho_{0}^{n}(h)-\int_{0}^{t} G \rho_{s}^{n}(h) \mathrm{d} s .
$$

From Theorem 3.1 in [18, page 28] and the arguments in the proof of Lemma 4, it is sufficient to show the following two conditions:

(A) $\sup _{s \leq t}\left|M_{s}^{n}(h)-N_{s}^{n}(h)\right| \stackrel{p}{\rightarrow} 0$ as $n \rightarrow \infty$, for each $t \geq 0$,

(B) $N^{n}(h) \stackrel{d}{\rightarrow} M(h)$ as $n \rightarrow \infty$.

To show (A), note that

$$
\begin{aligned}
& \sup _{s \leq t} \mid M_{s}^{n}(h)- N_{s}^{n}(h) \mid \\
&=\int_{0}^{t} \mid \int_{S} \lambda h(\mu, \lambda) f\left(\int_{S} \rho_{s}^{n}\left(\mathrm{~d} \mu^{\prime}, \mathrm{d} \lambda^{\prime}\right)+n^{-1} \sum_{j=1}^{n} g_{j}(0)\right) \\
& \quad \times\left(\sigma_{s}^{n}(\mathrm{~d} \mu, \mathrm{d} \lambda)-\sigma_{s}(\mathrm{~d} \mu, \mathrm{d} \lambda)\right) \mid \mathrm{d} s .
\end{aligned}
$$

From the argument following (17), there is some constant $C>$ 0 satisfying

$$
f\left(\int_{S} \rho_{s}^{n}\left(\mathrm{~d} \mu^{\prime}, \mathrm{d} \lambda^{\prime}\right)+n^{-1} \sum_{j=1}^{n} g_{j}(0)\right) \leq C .
$$

Hence, by the dominated convergence theorem,

$$
\begin{aligned}
& \sup _{s \leq t}\left|M_{s}^{n}(h)-N_{s}^{n}(h)\right| \\
& \leq C \int_{0}^{t}\left|\int_{S} \lambda h(\mu, \lambda)\left(\sigma_{s}^{n}(\mathrm{~d} \mu, \mathrm{d} \lambda)-\sigma_{s}(\mathrm{~d} \mu, \mathrm{d} \lambda)\right)\right| \mathrm{d} s \stackrel{p}{\longrightarrow} 0,
\end{aligned}
$$

as $n \rightarrow \infty$, since $\lambda h \in C_{b}(S)$ and the assumption (A2) holds.

To show (B), it is sufficient to show that [18, Theorem 2.1, page 16]

$$
E\left(l\left(N^{n}(h)\right)\right) \longrightarrow E(l(M(h))),
$$

as $n \rightarrow \infty$, for any bounded, uniformly continuous function $l: D\left(\mathbb{R}_{+}, \mathbb{R}\right) \rightarrow \mathbb{R}$. By the definition of $N^{n}(h)$ and the assumption $\rho^{n} \stackrel{d}{\rightarrow} \rho$, we only need to show that $l\left(N^{n}(h)\right)$ is a continuous function of $\rho^{n}$ mapping $D\left(\mathbb{R}_{+}, \Omega\right)$ to $\mathbb{R}$, invoking the dominated convergence theorem. Furthermore, it is sufficient to show that $N^{n}(h)$ is a continuous function of $\rho^{n}$ mapping $D\left(\mathbb{R}_{+}, \Omega\right)$ to $D\left(\mathbb{R}_{+}, \mathbb{R}\right)$. In the following, we take one term in $N^{n}(h)$ as an example to show the continuity with respect to $\rho^{n}$. Other terms can be shownanalogously. 
Define

$$
\begin{aligned}
l_{t}^{n} & :=\int_{S} \lambda h(\mu, \lambda) f\left(\int_{S} \rho_{t}^{n}\left(\mathrm{~d} \mu^{\prime}, \mathrm{d} \lambda^{\prime}\right)+\bar{g}\right) \rho_{t}^{n}(\mathrm{~d} \mu, \mathrm{d} \lambda), \\
l_{t} & :=\int_{S} \lambda h(\mu, \lambda) f\left(\int_{S} \rho_{t}\left(\mathrm{~d} \mu^{\prime}, \mathrm{d} \lambda^{\prime}\right)+\bar{g}\right) \rho_{t}(\mathrm{~d} \mu, \mathrm{d} \lambda) .
\end{aligned}
$$

Suppose that $\rho^{n} \rightarrow \rho$ in $D\left(\mathbb{R}_{+}, \Omega\right)$ holds. We need to show $\int_{0}^{t} l_{s}^{n} \mathrm{~d} s \rightarrow \int_{0}^{t} l_{s} \mathrm{~d} s$ in $D\left(\mathbb{R}_{+}, \mathbb{R}\right)$ as $n \rightarrow \infty$. Indeed, since $\lambda h \in C_{b}(S)$ and $\int_{S} h(\mu, \lambda) \rho_{t}(\mathrm{~d} \mu, \mathrm{d} \lambda): D\left(\mathrm{R}_{+}, \Omega\right) \rightarrow$ $D\left(\mathbb{R}_{+}, \mathbb{R}\right)$ is continuous for any $h \in C_{b}(S)$, we have $\int_{S} \lambda h(\mu, \lambda) \rho_{t}^{n}(\mathrm{~d} \mu, \mathrm{d} \lambda) \rightarrow \int_{S} \lambda h(\mu, \lambda) \rho_{t}(\mathrm{~d} \mu, \mathrm{d} \lambda)$ in $D\left(\mathbb{R}_{+}, \mathbb{R}\right)$ and $\int_{S} \rho_{t}^{n}(\mathrm{~d} \mu, \mathrm{d} \lambda) \rightarrow \int_{S} \rho_{t}(\mathrm{~d} \mu, \mathrm{d} \lambda)$ in $D\left(\mathbb{R}_{+}, \mathbb{R}\right)$, as $n \rightarrow$ $\infty$. Hence, from the assumptions (A3) and (A4), we obtain $l_{t}^{n} \rightarrow l_{t}$ in $D\left(\mathbb{R}_{+}, \mathbb{R}\right)$. It follows from [18, Equation (12.14), page 124] that $l_{t}^{n} \rightarrow l_{t}$ for all but countably many $t$. The dominated convergence theorem then yields $\int_{0}^{t} l_{s}^{n} \mathrm{~d} s \rightarrow$ $\int_{0}^{t} l_{s} \mathrm{~d} s$ in supremum norm on finite time intervals and hence in $D\left(\mathrm{R}_{+}, \mathrm{R}\right)$.

Lemma 6. The solution to (5) is unique in $D\left(\mathbb{R}_{+}, \Omega\right)$.

Proof. Suppose that $\rho$ and $\widetilde{\rho}$ are two solutions to (5), and they are the limits of two weakly convergent subsequences of $\rho^{n}$, respectively. By the assumption (A2), $\rho_{0}=\widetilde{\rho}_{0}$. We need to show that $\rho_{t}=\tilde{\rho}_{t}$ for all $t \in \mathbb{R}_{+}$.

From (3), we have

$$
\int_{S} h(\mu, \lambda) \rho_{t}^{n}(\mathrm{~d} \mu, \mathrm{d} \lambda) \leq \int_{S} h(\mu, \lambda) \sigma_{t}^{n}(\mathrm{~d} \mu, \mathrm{d} \lambda),
$$

for any $h \in C_{b}(S)$. Therefore, by the assumption (A2),

$$
\begin{gathered}
\int_{S} h(\mu, \lambda) \rho_{t}(\mathrm{~d} \mu, \mathrm{d} \lambda) \leq \int_{S} h(\mu, \lambda) \sigma_{t}(\mathrm{~d} \mu, \mathrm{d} \lambda) \\
\int_{S} h(\mu, \lambda) \tilde{\rho}_{t}(\mathrm{~d} \mu, \mathrm{d} \lambda) \leq \int_{S} h(\mu, \lambda) \sigma_{t}(\mathrm{~d} \mu, \mathrm{d} \lambda)
\end{gathered}
$$

hold. Define $d\left(\rho_{t}, \tilde{\rho}_{t}\right)=\sup _{|h| \leq 1, h \in C_{b}(S)}\left|\rho_{t}(h)-\tilde{\rho}_{t}(h)\right|$. In view of (5), we obtain

$$
\begin{aligned}
& \left|\rho_{t}(h)-\widetilde{\rho}_{t}(h)\right| \\
& =\int_{0}^{t} G \rho_{s}(h)-G \widetilde{\rho}_{s}(h) \mathrm{d} s \\
& \leq \int_{0}^{t} \int_{S} \lambda h(\mu, \lambda) \mid f\left(\int_{S} \rho_{s}\left(\mathrm{~d} \mu^{\prime}, \mathrm{d} \lambda^{\prime}\right)+\bar{g}\right) \\
& \quad-f\left(\int_{S} \tilde{\rho}_{s}\left(\mathrm{~d} \mu^{\prime}, \mathrm{d} \lambda^{\prime}\right)+\bar{g}\right) \mid \\
& +\int_{0}^{t} \int_{S} \lambda h(\mu, \lambda) \mid f\left(\int_{S} \rho_{s}\left(\mathrm{~d} \mu^{\prime}, \mathrm{d} \lambda^{\prime}\right)+\bar{g}\right) \\
& \quad-f\left(\int_{S} \tilde{\rho}_{s}\left(\mathrm{~d} \mu^{\prime}, \mathrm{d} \lambda^{\prime}\right)+\bar{g}\right) \mid \\
& \quad \times \rho_{s}(\mathrm{~d} \mu, \mathrm{d} \lambda) \mathrm{d} s
\end{aligned}
$$

$$
\begin{aligned}
& +\int_{0}^{t}\left|\int_{S} \lambda h(\mu, \lambda) \rho_{s}(\mathrm{~d} \mu, \mathrm{d} \lambda)-\int_{S} \lambda h(\mu, \lambda) \tilde{\rho}_{s}(\mathrm{~d} \mu, \mathrm{d} \lambda)\right| \\
& \quad \times f\left(\int_{S} \tilde{\rho}_{s}\left(\mathrm{~d} \mu^{\prime}, \mathrm{d} \lambda^{\prime}\right)+\bar{g}\right) \mathrm{d} s \\
& +\int_{0}^{t} \mid \int_{S} \mu h(\mu, \lambda) \rho_{s}(\mathrm{~d} \mu, \mathrm{d} \lambda) \\
& \quad-\int_{S} \mu h(\mu, \lambda) \tilde{\rho}_{s}(\mathrm{~d} \mu, \mathrm{d} \lambda) \mid \mathrm{d} s .
\end{aligned}
$$

The assumption (A4) indicates that

$$
\begin{aligned}
& \left|f\left(\int_{S} \rho_{s}\left(\mathrm{~d} \mu^{\prime}, \mathrm{d} \lambda^{\prime}\right)+\bar{g}\right)-f\left(\int_{S} \tilde{\rho}_{s}\left(\mathrm{~d} \mu^{\prime}, \mathrm{d} \lambda^{\prime}\right)+\bar{g}\right)\right| \\
& \quad \leq C_{f}\left|\int_{S} \rho_{s}\left(\mathrm{~d} \mu^{\prime}, \mathrm{d} \lambda^{\prime}\right)-\int_{S} \tilde{\rho}_{s}\left(\mathrm{~d} \mu^{\prime}, \mathrm{d} \lambda^{\prime}\right)\right| \\
& \quad \leq C_{f} d\left(\rho_{s}, \tilde{\rho}_{s}\right)
\end{aligned}
$$

for some constant $C_{f}>0$. Therefore, using (38) we can derive

$$
\begin{aligned}
& \left|\rho_{t}(h)-\tilde{\rho}_{t}(h)\right| \\
& \leq 2 C_{f} \int_{0}^{t}\left(\int_{S} \lambda h(\mu, \lambda) \sigma_{s}(\mathrm{~d} \mu, \mathrm{d} \lambda)\right) d\left(\rho_{s}, \widetilde{\rho}_{s}\right) \mathrm{d} s \\
& \quad+C\left(\sup _{(\mu, \lambda) \in S} \lambda\right) \int_{0}^{t} d\left(\rho_{s}, \widetilde{\rho}_{s}\right) \mathrm{d} s \\
& \quad+\left(\sup _{(\mu, \lambda) \in S} \mu\right) \int_{0}^{t} d\left(\rho_{s}, \widetilde{\rho}_{s}\right) \mathrm{d} s,
\end{aligned}
$$

where $C>0$ is some constant. To see this, note that by the assumptions (A1), (A2), and (38), we have

$$
\int_{S} \tilde{\rho}_{s}\left(\mathrm{~d} \mu^{\prime}, \mathrm{d} \lambda^{\prime}\right)+\bar{g} \leq \int_{S} \sigma_{s}\left(\mathrm{~d} \mu^{\prime}, \mathrm{d} \lambda^{\prime}\right)+\bar{g}<\infty,
$$

for any $0 \leq s \leq t$. The assumption (A4) then implies

$$
\sup _{s \leq t} f\left(\int_{S} \tilde{\rho}_{s}\left(\mathrm{~d} \mu^{\prime}, \mathrm{d} \lambda^{\prime}\right)+\bar{g}\right) \leq C<\infty
$$

Again employing the assumption (A2), we have $\int_{S} \lambda h(\mu$, $\lambda) \sigma_{s}(\mathrm{~d} \mu, \mathrm{d} \lambda)<\infty$ for all $0 \leq s \leq t$. Hence, by using the assumption (A3), the inequality (42) becomes

$$
d\left(\rho_{s}, \tilde{\rho}_{s}\right) \leq C^{\prime} \int_{0}^{t} d\left(\rho_{s}, \tilde{\rho}_{s}\right) \mathrm{d} s
$$

for some constant $C^{\prime}>0$. A simple application of Gronwall's lemma yields $d\left(\rho_{t}, \widetilde{\rho}_{t}\right)=0$ for all $t \in \mathbb{R}_{+}$, which concludes the proof.

Proof of Theorem 1. By Lemma 4 and Lemma 5, the limit of any weakly convergent subsequence of $\rho^{n}$ must satisfy (5). By Lemma 6 , we find that the sequence $\rho^{n}$ must itself converge weakly to that unique solution (see the corollary in [18, page 59]). The proof of Theorem 1 is thus completed. 
Proof of Corollary 2. For any open set $F \subseteq S$, we obtain from (38) that

$$
\int_{S} f^{n}(\mu, \lambda) \rho_{t}(\mathrm{~d} \mu, \mathrm{d} \lambda) \leq \int_{S} f^{n}(\mu, \lambda) \sigma_{t}(\mathrm{~d} \mu, \mathrm{d} \lambda)
$$

where $f^{n}$ is taken as a continuous function upwardly converging to the indicator function of $F$. By using the dominated convergence theorem, we know that $\rho_{t}(F) \leq \sigma_{t}(F)$. A regularity property (see, e.g., [17, page 18 , Lemma 1.34]) implies that $\rho_{t}(A) \leq \sigma_{t}(A)$ for all $A \in S$. This means that $\rho_{t}$ is absolutely continuous with respect to $\sigma_{t}$ for any $t \geq 0$. An application of the Radon-Nykodym theorem yields the existence of $\varphi_{t}$ such that (8) holds with $0 \leq \varphi_{t} \leq 1$. Now the result follows straightforwardly from Theorem 1 .

\section{Acknowledgments}

The author expresses his sincere gratitude to the anonymous referee and the editor for careful reading of the original paper and useful comments that helped to improve presentation of results.

\section{References}

[1] G. H. Weiss and M. Dishon, "On the asymptotic behavior of the stochastic and deterministic models of an epidemic," Mathematical Biosciences, vol. 11, pp. 261-265, 1971.

[2] D. A. Kessler, "Epidemic size in the SIS model of endemic infections," Journal of Applied Probability, vol. 45, no. 3, pp. 757778, 2008.

[3] Y. Shang, "A Lie algebra approach to susceptible-infectedsusceptible epidemics," Electronic Journal of Differential Equations, vol. 2012, no. 233, pp. 1-7, 2012.

[4] H. Andersson and B. Djehiche, "A threshold limit theorem for the stochastic logistic epidemic," Journal of Applied Probability, vol. 35, no. 3, pp. 662-670, 1998.

[5] C. R. Doering, K. V. Sargsyan, and L. M. Sander, "Extinction times for birth-death processes: exact results, continuum asymptotics, and the failure of the Fokker-Planck approximation," Multiscale Modeling \& Simulation, vol. 3, no. 2, pp. 283299, 2005.

[6] I. Nåsell, "On the quasi-stationary distribution of the stochastic logistic epidemic," Mathematical Biosciences, vol. 156, no. 1-2, pp. 21-40, 1999.

[7] O. Ovaskainen, "The quasistationary distribution of the stochastic logistic model," Journal of Applied Probability, vol. 38, no. 4, pp. 898-907, 2001.

[8] D. Clancy and P. K. Pollett, "A note on quasi-stationary distributions of birth-death processes and the SIS logistic epidemic," Journal of Applied Probability, vol. 40, no. 3, pp. 821-825, 2003.

[9] T. G. Kurtz, "Extensions of Trotter's operator semigroup approximation theorems," Journal of Functional Analysis, vol. 3, pp. 354-375, 1969.

[10] T. G. Kurtz, "Solutions of ordinary differential equations as limits of pure jump Markov processes," Journal of Applied Probability, vol. 7, pp. 49-58, 1970.

[11] R. McVinish and P. K. Pollett, "The deterministic limit of a stochastic logistic model with individual variation," Mathematical Biosciences, vol. 241, no. 1, pp. 109-114, 2013.
[12] R. W. R. Darling and J. R. Norris, "Differential equation approximations for Markov chains," Probability Surveys, vol. 5, pp. 37-79, 2008.

[13] Y. Shang, "Optimal control strategies for virus spreading in inhomogeneousepidemicdynamics," Canadian Mathematical Bulletin. In press.

[14] T. Gross, C. J. D. D’Lima, and B. Blasius, "Epidemic dynamics on an adaptivenetwork," Physical Review Letters, vol. 96, no. 20, Article ID 208701, 2006.

[15] Y. Shang, "Discrete-time epidemic dynamics with awareness in random networks," International Journal of Biomathematics, vol. 6, no. 2, Article ID 1350007, 2013.

[16] K. Klemm, M. Á. Serrano, V. M. Eguíluz, and M. S. Miguel, "A measure of individualrole in collective dynamics," Scientific Reports, vol. 2, article 292, 2012.

[17] O. Kallenberg, Foundations of Modern Probability, Springer, New York, NY, USA, 2nd edition, 2002.

[18] P. Billingsley, Convergence of Probability Measures, John Wiley \& Sons, New York, NY, USA, 2nd edition, 1999.

[19] S. N. Ethier and T. G. Kurtz, Markov Processes: Characterization and Convergence, John Wiley \& Sons, Hoboken, NJ, USA, 1986. 


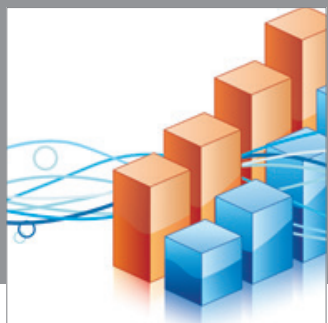

Advances in

Operations Research

mansans

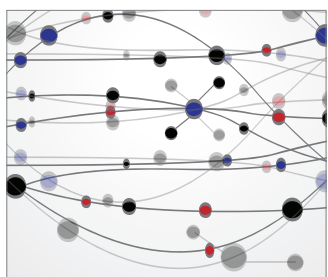

The Scientific World Journal
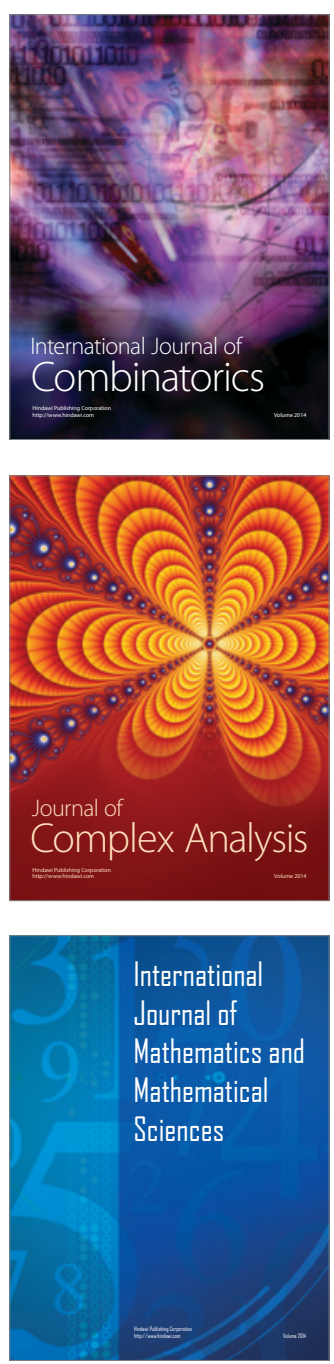
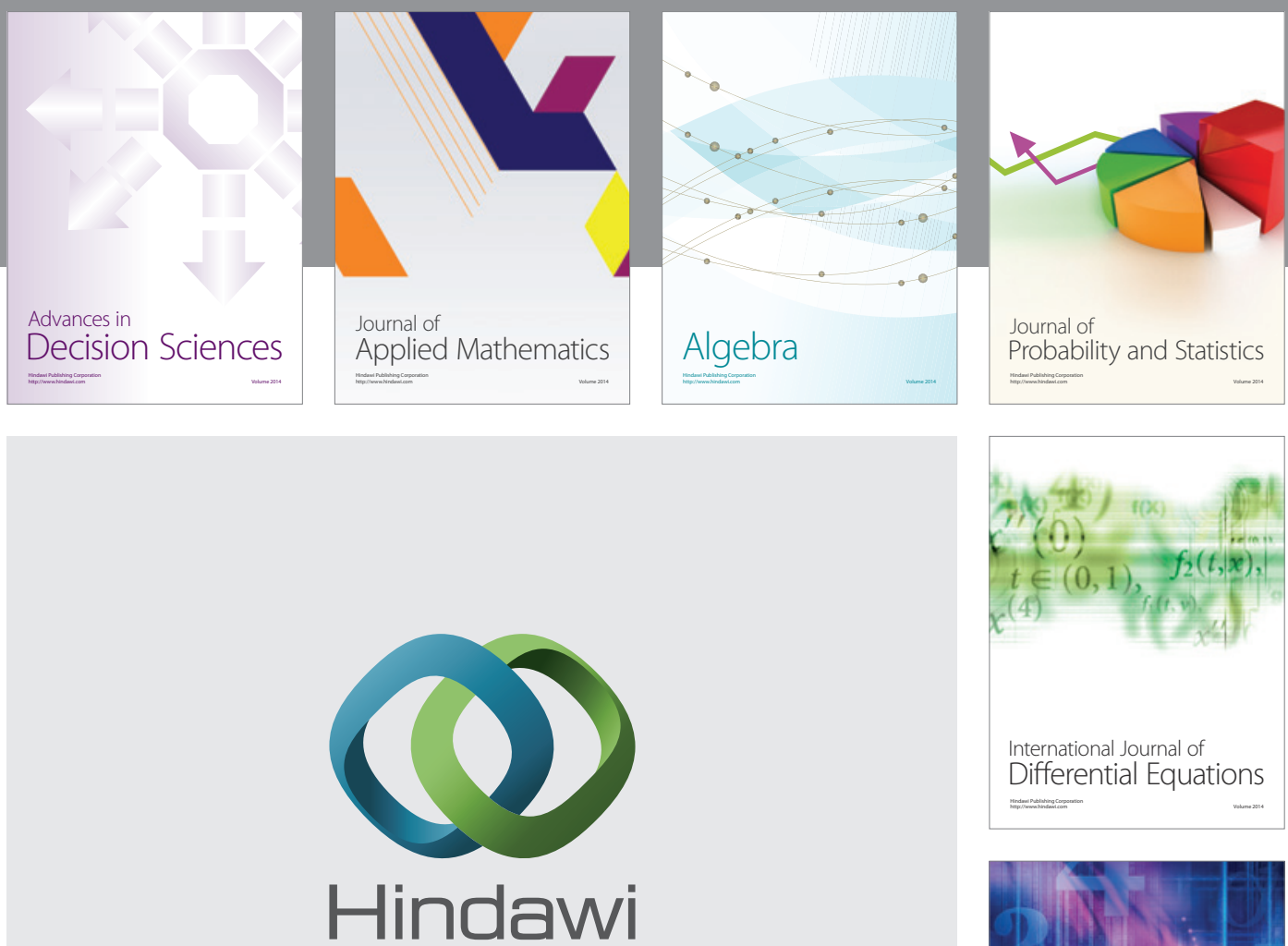

Submit your manuscripts at http://www.hindawi.com
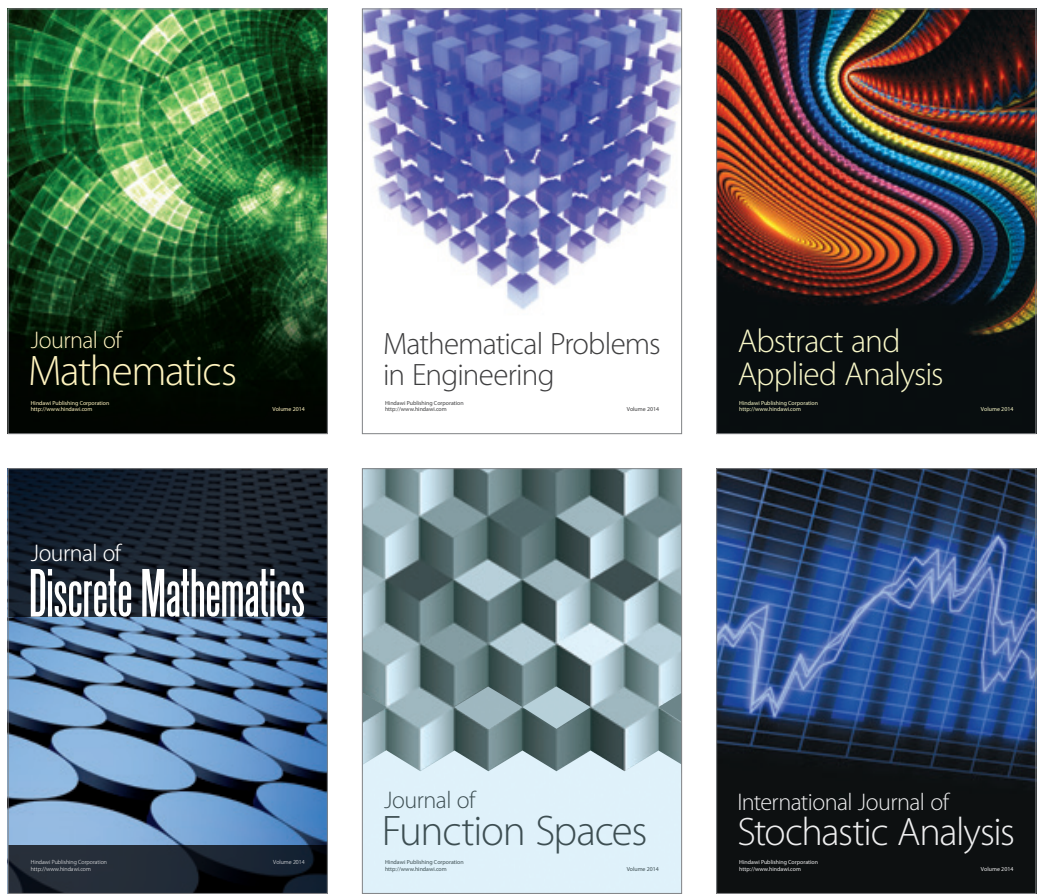

Journal of

Function Spaces

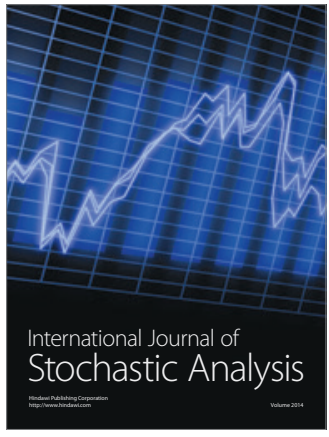

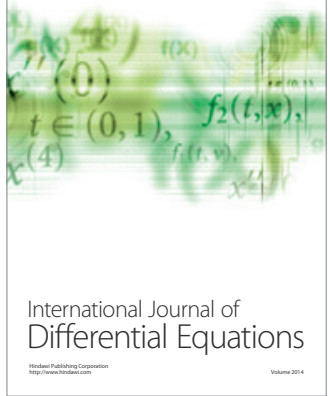
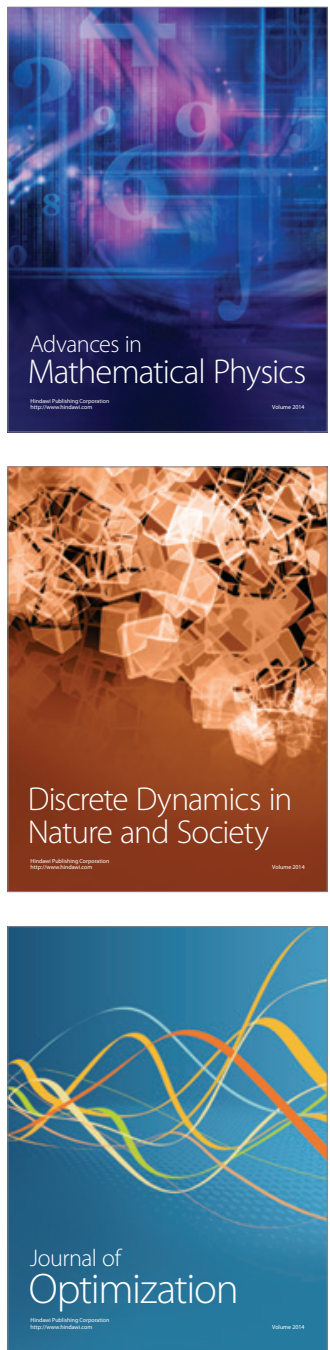